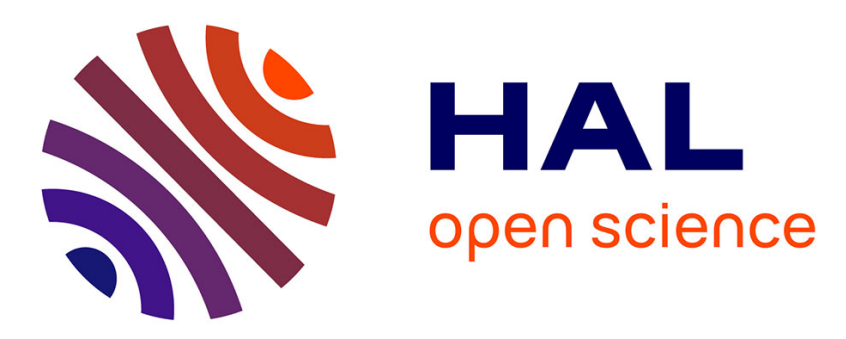

\title{
CRLB under K-distributed observation with parameterized mean
}

\author{
Mohammed Nabil El Korso, Alexandre Renaux, Philippe Forster
}

\section{To cite this version:}

Mohammed Nabil El Korso, Alexandre Renaux, Philippe Forster. CRLB under K-distributed observation with parameterized mean. IEEE Sensor Array Multichannel Workshop, Jun 2014, A Coruña, Spain. hal-00993010

\section{HAL Id: hal-00993010 https://hal.inria.fr/hal-00993010}

Submitted on 19 May 2014

HAL is a multi-disciplinary open access archive for the deposit and dissemination of scientific research documents, whether they are published or not. The documents may come from teaching and research institutions in France or abroad, or from public or private research centers.
L'archive ouverte pluridisciplinaire HAL, est destinée au dépôt et à la diffusion de documents scientifiques de niveau recherche, publiés ou non, émanant des établissements d'enseignement et de recherche français ou étrangers, des laboratoires publics ou privés. 


\title{
CRLB UNDER K-DISTRIBUTED OBSERVATION WITH PARAMETERIZED MEAN
}

\author{
Mohammed Nabil EL KORSO, Alexandre RENAUX and Philippe FORSTER \\ Université Paris-Ouest X, IUT de Ville d'Avray, LEME EA 4416, France, m.elkorso@u-paris10.fr \\ Université Paris-Sud XI, L2S UMR 8506, France, alexandre.renaux@u-psud.fr \\ Université Paris-Ouest X, SATIE UMR 8029, France, pforster@u-paris10.fr
}

\begin{abstract}
A semi closed-form expression of the Fisher information matrix in the context of K-distributed observations with parameterized mean is given and related to the classical, i.e. Gaussian case. This connection is done via a simple multiplicative factor, which only depends on the intrinsic parameters of the texture and the size of the observation vector. Finally, numerical simulation is provided to corroborate the theoretical analysis.
\end{abstract}

\section{INTRODUCTION}

The Cramér-Rao lower bound (CRLB) is well known to be a popular tool in order to study performance, in terms of variance, of unbiased estimators. This comes from the fact that, for several observation models, it can be achieved asymptotically when the number of observation is large [1] or when the signal-to-noise ratio (SNR) is high [2]. Moreover, in the specific but widely, used case of Gaussian observations with parameterized mean and/or covariance matrix, it can be computed very easily from the so-called Slepian-Bang formula [3]. However, when the observations are non-Gaussian, the Slepian-Bang formula does not apply and the derivations of the CRLB has to be analyzed specifically.

Among non-Gaussian probability density functions, the spherically invariant random vectors (SIRV) are known to fit with noise/clutter met in real applications [4]. Various SIRV have been studied in the past with several applications, e.g., radar detection [5]. In this communication, we are interested to a particular SIRV named K-distribution. A complex Kdistribution can be seen as the product of a complex circular Gaussian random vector, with zero mean and covariance matrix $\mathbf{M}$, and the square root of a gamma distributed random variable independent of this vector. The applications of such random vectors are large especially in radar signal processing [6].

Of course, the Slepian-Bang formula does not apply when the observation vector is K-distributed. Several authors have consequently studied the CRLB for such problems. One can cite [7]-[10] where the parameter of interest is the covariance

This research was supported by the iCODE institute (research project of the IDEX Paris-Saclay), the NEWCOM $\sharp$, the MASTODONS project and the DGA/DGCIS. matrix M. In the context of K-distributed observations with parameterized mean, the hybrid CRLB has been studied in [8] in the context of spectral analysis by way of Monte-Carlo simulation. In [11], [12], the true CRLB has been derived w.r.t. the signal amplitudes in the context of array processing. In this paper, we propose a general formula of the true CRLB whatever the kind of parameterization. Finally, with the aid of numerical tools, we provide a detailed discussion on several enlightening properties of the CRLB revealed by our expression, with an emphasis on the relationship with the classical Gaussian case.

We advice the reader to consult reference [13], in which the authors proposed a general compact semi-closed form expression for the CRLB under the so-called multivariate elliptically contoured distributed observations. Such distribution is of great interest since it includes a wide kind of distributions among them the K-distribution. Unfortunately, for K-distributed observations, the proposed expression in [13] has not been studied. Consequently, in this communication, we propose another way to derive the CRLB for only the specific case of the K-distributed observations scenario.

\section{OBSERVATION MODEL}

In the sequel, we consider the following general observation model with parameterized mean

$$
\mathbf{x}(t)=\mathbf{m}(\theta, t)+\mathbf{n}(t), \quad t=1, \ldots, L,
$$

where $t$ denotes the $t$-th observation, the observation vector $\mathbf{x}(t) \in \mathbb{C}^{N}$ is statistically independent from observation to observation, the additive noise vector $\mathbf{n}(t)$ is modelled as a complex multivariate $\mathrm{K}$-distributed process. $\mathbf{m}(\theta, t)$ is a given deterministic known function (possibly nonlinear) and assumed twice derivable w.r.t. $\theta \in \mathbb{R}$ which is the parameter of interest.

From the spherically invariant random vector definition [14], one can decompose the multivariate K-distributed process as the product of two components: $\tau(t)$ which is the so-called texture term, and $\mathbf{c}(t)$ which is the speckle term, one has:

$$
\mathbf{n}(t)=\sqrt{\tau(t)} \mathbf{c}(t),
$$

in which $\tau(t)$ is a gamma distributed process with parameters $\alpha$ and $\frac{4}{\beta^{2}}$, i.e., $\tau(t) \sim \mathcal{G}\left(\alpha, \frac{4}{\beta^{2}}\right)$, whereas, $\mathbf{c}(t)$ denotes a complex circular zero-mean Gaussian distributed process with covariance matrix $\mathbf{M}$, i.e., $\mathbf{c}(t) \sim \mathcal{C N}(\mathbf{0}, \mathbf{M})$. 
We can note that the parameter $\alpha$ affects the spikiness of the additive noise. More precisely, for small $\alpha$, the noise $\mathbf{n}(t)$ becomes heavier, whereas, for high $\alpha$, the noise tends to be Gaussian. Let us define $z(\theta, t)=$ $(\mathbf{x}(t)-\mathbf{m}(\theta, t))^{H} \mathbf{M}^{-1}(\mathbf{x}(t)-\mathbf{m}(\theta, t))$.Consequently, the probability function of the observation vector is given by

$f(\mathbf{x}(t) ; \theta, \mathbf{M})=\frac{\beta^{\alpha+N} z(\theta, t)^{\frac{\alpha-N}{2}}}{2^{\alpha+N-1} \pi^{N}|\mathbf{M}| \Gamma(\alpha)} K_{N-\alpha}(\beta \sqrt{z(\theta, t)})$

in which $K_{\mu}($.$) and \Gamma($.$) denote, respectively, the modified$ Bessel function of the second kind of order $\mu$ and the gamma function [15].

\section{CRAMÉR-RAO BOUND DERIVATION}

Let $\mathbb{E}\left\{(\hat{\theta}-\theta)^{2}\right\}$ be the variance of an unbiased estimator of $\theta$, denoted by $\hat{\theta}$. The Cramér-Rao inequality states that $\mathbb{E}\left\{(\hat{\theta}-\theta)^{2}\right\} \geq \operatorname{CRLB}(\theta)=\left[\operatorname{FIM}(\boldsymbol{\xi})^{-1}\right]_{1,1}$ where $\boldsymbol{\xi}=\left[\theta, \mathcal{S}(\mathbf{M})^{T}\right]^{T}$ in which the operator $\mathcal{S}(\mathbf{M})$ stacks the real part of the upper triangular portion and stacks the imaginary part of the upper triangular portion excluding the diagonal of the matrix $\mathbf{M}$. It has been proved in [13] that $\mathbf{F I M}(\boldsymbol{\xi})=\operatorname{diag}\{\operatorname{FI}(\theta), \mathbf{F I}(\mathcal{S}(\mathbf{M}))\}$. Consequently, $\operatorname{CRLB}(\theta)=\frac{1}{\operatorname{FI}(\theta)}$ where, for independent observations, the latter simplifies to $\operatorname{FI}(\theta)=-\sum_{t=1}^{L} \mathbb{E}\left\{\frac{d^{2} \ln f(\mathbf{x}(t) ; \theta)}{d \theta^{2}}\right\}$ in which for sake of notation simplicity, the observation probability density function is denoted by $f(\mathbf{x}(t) ; \theta)$. Note that $\mathbf{F I}(\mathcal{S}(\mathbf{M}))$ in the context of K-distributed clutter can be found in [9] but that $\operatorname{CRLB}(\theta)$ has not been studied before.

\section{III-A. Score function and its derivative}

First, let us rewrite $\frac{d^{2} \ln f(\mathbf{x}(t) ; \theta)}{d \theta^{2}}$ as follows

$$
\begin{aligned}
& \frac{d^{2} \ln f(\mathbf{x}(t) ; \theta)}{d \theta^{2}}=\frac{d}{d \theta} \frac{d \ln f(\mathbf{x}(t) ; \theta)}{d z(\theta, t)} \frac{d z(\theta, t)}{d \theta} \\
& =\frac{d}{d z(\theta, t)}\left[\frac{d \ln f(\mathbf{x}(t) ; \theta)}{d z(\theta, t)} \frac{d z(\theta, t)}{d \theta}\right] \frac{d z(\theta, t)}{d \theta} \\
& +\frac{d \ln f(\mathbf{x}(t) ; \theta)}{d z(\theta, t)} \frac{d^{2} z(\theta, t)}{d \theta^{2}} \\
& =\frac{d \frac{1}{f(\mathbf{x}(t) ; \theta)} \frac{d f(\mathbf{x}(t) ; \theta)}{d z(\theta, t)}\left(\frac{d z(\theta, t)}{d \theta}\right)^{2}}{+\frac{1}{f(\mathbf{x}(t) ; \theta)} \frac{d f(\mathbf{x}(t) ; \theta)}{d z(\theta, t)} \frac{d^{2} z(\theta, t)}{d \theta^{2}}} \\
& =\frac{1}{f(\mathbf{x}(t) ; \theta)} \frac{d^{2} f(\mathbf{x}(t) ; \theta)}{d z(\theta, t)^{2}}\left(\frac{d z(\theta, t)}{d \theta}\right)^{2} \\
& +\frac{1}{f(\mathbf{x}(t) ; \theta)} \frac{d f(\mathbf{x}(t) ; \theta)}{d z(\theta, t)} \frac{d^{2} z(\theta, t)}{d \theta^{2}} \\
& -\frac{1}{f(\mathbf{x}(t) ; \theta)^{2}}\left(\frac{d f(\mathbf{x}(t) ; \theta)}{d z(\theta, t)}\right)^{2}\left(\frac{d z(\theta, t)}{d \theta}\right)^{2} .
\end{aligned} .
$$

Thus, let us detail each component involved in (4), i.e., $\frac{d z(\theta, t)}{d \theta}, \frac{d^{2} z(\theta, t)}{d \theta^{2}}, \frac{d f(\mathbf{x}(t) ; \theta)}{d z(\theta, t)}$ and $\frac{d^{2} f(\mathbf{x}(t) ; \theta)}{d z(\theta, t)^{2}}$

Trivially, one has

$$
\frac{d z(\theta, t)}{d \theta}=2 \Re\left\{(\mathbf{m}(\theta, t)-\mathbf{x}(t))^{H} \mathbf{M}^{-1} \frac{d \mathbf{m}(\theta, t)}{d \theta}\right\},
$$

and $\frac{d^{2} z(\theta, t)}{d \theta^{2}}=2 \Re\left\{(\mathbf{m}(\theta, t)-\mathbf{x}(t))^{H} \mathbf{M}^{-1} \frac{d^{2} \mathbf{m}(\theta, t)}{d \theta^{2}}\right\}$

$$
+2 \frac{d \mathbf{m}(\theta, t)^{H}}{d \theta} \mathbf{M}^{-1} \frac{d \mathbf{m}(\theta, t)}{d \theta} .
$$

To obtain $\frac{d f(\mathbf{x}(t) ; \theta)}{d z(\theta, t)}$ and $\frac{d^{2} f(\mathbf{x}(t) ; \theta)}{d z(\theta, t)^{2}}$, let us set

$$
\nu=\frac{\beta^{\alpha+N}}{2^{\alpha+N-1} \pi^{N}|\mathbf{M}| \Gamma(\alpha)}
$$

by the fact that $\frac{d K_{\mu}(\varrho)}{d \varrho}=-K_{\mu+1}(\varrho)+\frac{\mu}{\varrho} K_{\mu}(\varrho)$ one has

$$
\begin{aligned}
\frac{d K_{N-\alpha}(\beta \sqrt{z(\theta, t)})}{d z(\theta, t)}= & \frac{-\beta}{2 \sqrt{z(\theta, t)}} K_{N-\alpha+1}(\beta \sqrt{z(\theta, t)}) \\
& +\frac{N-\alpha}{2 z(\theta, t)} K_{N-\alpha}(\beta \sqrt{z(\theta, t)})
\end{aligned}
$$

Consequently,

$$
\frac{d f(\mathbf{x}(t) ; \theta)}{d z(\theta, t)}=-\frac{\beta \nu}{2} z(\theta, t)^{\frac{\alpha-N}{2}-\frac{1}{2}} K_{N-\alpha+1}(\beta \sqrt{z(\theta, t)}) .
$$

Finally, using (8) and (7), one obtains

$$
\frac{d^{2} f(\mathbf{x}(t) ; \theta)}{d z(\theta, t)^{2}}=\frac{\nu \beta^{2}}{4} z(\theta, t)^{\frac{\alpha-N}{2}-1} K_{N-\alpha+2}(\beta \sqrt{z(\theta, t)}) .
$$

\section{III-B. Fisher information derivation}

In the following, we derive the Fisher information given by $\operatorname{FI}(\theta)=-\sum_{t=1}^{L} \mathbb{E}\left\{\frac{d^{2} \ln f(\mathbf{x}(t) ; \theta)}{d \theta^{2}}\right\}$.

First, let us derive the first term's expectation of (4). Plugging (3), (5) and (9) into (4) and using the expectation operator, one obtains

$$
\begin{aligned}
& \mathbb{E}\left\{\frac{1}{f(\mathbf{x}(t) ; \theta)} \frac{d^{2} f(\mathbf{x}(t) ; \theta)}{d z(\theta, t)^{2}}\left(\frac{d z(\theta, t)}{d \theta}\right)^{2}\right\}= \\
& \frac{\beta^{4}}{4} \frac{\Gamma(\alpha-2)}{\Gamma(\alpha)} \mathbb{E}\left\{\Re^{2}\left\{(\mathbf{m}(\theta, t)-\mathbf{x}(t))^{H} \mathbf{M}^{-1} \frac{d \mathbf{m}(\theta, t)}{d \theta}\right\}\right\},
\end{aligned}
$$

where the expectation is taken with respect to the following probability density function

$f(\mathbf{x}(t) ; \theta)=\frac{\beta^{\alpha+N-2} z(\theta, t)^{\frac{\alpha-N-2}{2}}}{2^{\alpha+N-3} \pi^{N}|\mathbf{M}| \Gamma(\alpha-2)} K_{N-\alpha+2}(\beta \sqrt{z(\theta, t)})$, 
which is a complex K-distribution $\mathcal{K}_{N}\left(\alpha-2, \frac{4}{\beta^{2}}, \mathbf{M}\right)$. Using the SIRV decomposition given by (2) and the independence between $\tau(t)$ and $\mathbf{c}(t),(10)$ becomes

$$
\begin{array}{r}
\mathbb{E}\left\{\frac{1}{f(\mathbf{x}(t) ; \theta)} \frac{d^{2} f(\mathbf{x}(t) ; \theta)}{d z(\theta, t)^{2}}\left(\frac{d z(\theta, t)}{d \theta}\right)^{2}\right\}= \\
\frac{\beta^{4}}{4} \frac{\Gamma(\alpha-2)}{\Gamma(\alpha)} \mathbb{E}\{\tau\} \mathbb{E}\left\{\tilde{c}(t)^{2}\right\}
\end{array}
$$

in which $\tilde{c}(t)=\Re\left\{\mathbf{c}(t)^{H} \mathbf{M}^{-1} \frac{d \mathbf{m}(\theta, t)}{d \theta}\right\}$. Since $\mathbf{c}(t) \sim \mathcal{C N}(\mathbf{0}, \mathbf{M})$, thus, $\mathbf{c}(t)^{H} \mathbf{M}^{-1} \frac{d \mathbf{m}(\theta, t)}{d \theta} \sim$ $\mathcal{C N}\left(\mathbf{0}, \frac{d \mathbf{m}(\theta, t)^{H}}{d \theta} \mathbf{M}^{-1} \frac{d \mathbf{m}(\theta, t)}{d \theta}\right)$. Furthermore, since $\mathbf{c}(t)$ is assumed to be circular, this implies that $\tilde{\mathbf{c}}(t) \sim$ $\mathcal{N}\left(\mathbf{0}, \frac{1}{2} \frac{d \mathbf{m}(\theta, t)^{H}}{d \theta} \mathbf{M}^{-1} \frac{d \mathbf{m}(\theta, t)}{d \theta}\right)$ and finally, (11) reduces to

$$
\begin{aligned}
& \frac{\beta^{4}}{8} \frac{\Gamma(\alpha-2)}{\Gamma(\alpha)} \mathbb{E}\{\tau\} \frac{d \mathbf{m}(\theta, t)^{H}}{d \theta} \mathbf{M}^{-1} \frac{d \mathbf{m}(\theta, t)}{d \theta}= \\
& \frac{\beta^{2}}{2}(\alpha-2) \frac{\Gamma(\alpha-2)}{\Gamma(\alpha)} \frac{d \mathbf{m}(\theta, t)^{H}}{d \theta} \mathbf{M}^{-1} \frac{d \mathbf{m}(\theta, t)}{d \theta}
\end{aligned}
$$

Second, let us derive the second term's expectation of (4). Plugging (3), (6) and (8) in (4), one obtains

$$
\begin{aligned}
& \mathbb{E}\left\{\frac{1}{f(\mathbf{x}(t) ; \theta)} \frac{d f(\mathbf{x}(t) ; \theta)}{d z(\theta, t)} \frac{d^{2} z(\theta, t)}{d \theta^{2}}\right\}= \\
& -\frac{\beta^{2}}{2} \frac{\Gamma(\alpha-1)}{\Gamma(\alpha)} \mathbb{E}\left\{\Re\left\{(\mathbf{m}(\theta, t)-\mathbf{x}(t))^{H} \mathbf{M}^{-1} \frac{d^{2} \mathbf{m}(\theta, t)}{d \theta^{2}}\right\}\right. \\
& \left.+\frac{d \mathbf{m}(\theta, t)^{H}}{d \theta} \mathbf{M}^{-1} \frac{d \mathbf{m}(\theta, t)}{d \theta}\right\},
\end{aligned}
$$

where the last expectation is taken with respect to following probability density function

$f(\mathbf{x}(t))=\frac{\beta^{\alpha+N-1} z(\theta, t)^{\frac{\alpha-N-1}{2}}}{2^{\alpha+N-2} \pi^{N}|\mathbf{M}| \Gamma(\alpha-1)} K_{N+1-\alpha}(\beta \sqrt{z(\theta, t)})$,

which is a complex K-distribution $\mathcal{K}_{N+1}\left(\alpha-1, \frac{4}{\beta^{2}}, \boldsymbol{M}\right)$. Using the SIRV decomposition given by (2) into (13) one deduces

$$
\begin{aligned}
& \mathbb{E}\left\{\frac{1}{f(\mathbf{x}(t) ; \theta)} \frac{d f(\mathbf{x}(t) ; \theta)}{d z(\theta, t)} \frac{d^{2} z(\theta, t)}{d \theta^{2}}\right\}= \\
& -\frac{\beta^{2}}{2} \frac{\Gamma(\alpha-1)}{\Gamma(\alpha)} \frac{d \mathbf{m}(\theta, t)^{H}}{d \theta} \mathbf{M}^{-1} \frac{d \mathbf{m}(\theta, t)}{d \theta}
\end{aligned}
$$

because $\Re\left\{\mathbf{c}(t)^{H} \mathbf{M}^{-1} \frac{d^{2} \mathbf{m}(\theta, t)}{d \theta^{2}}\right\}$ follows a Gaussian distribution with zero mean.
Finally, let us derive the third term's expectation of (4). Plugging (3), (5) and (8) in (4), one obtains

$$
\begin{aligned}
& \mathbb{E}\left\{\frac{1}{f(\mathbf{x}(t) ; \theta)^{2}}\left(\frac{d f(\mathbf{x}(t) ; \theta)}{d z(\theta, t)}\right)^{2}\left(\frac{d z(\theta, t)}{d \theta}\right)^{2}\right\}= \\
& \mathbb{E}\left\{\frac{\beta^{2}}{z(\theta, t)} \frac{K_{N-\alpha+1}(\beta \sqrt{z(\theta, t)})^{2}}{K_{N-\alpha}(\beta \sqrt{z(\theta, t)})^{2}} \Re^{2}\left\{\mathbf{n}(t)^{H} \mathbf{M}^{-1} \frac{d \mathbf{m}(\theta, t)}{d \theta}\right\}\right\} \\
& =\beta^{2} \mathbb{E}\left\{\frac{K_{N-\alpha+1}(\beta \sqrt{\tau(t)})^{2}}{K_{N-\alpha}(\beta \sqrt{\tau(t)})^{2}}\right\} \mathbb{E}\left\{\Re^{2}\left\{\mathbf{c}(t)^{H} \mathbf{M}^{-1} \frac{d \mathbf{m}(\theta, t)}{d \theta}\right\}\right\} \\
& =\frac{\beta^{2}}{2} \mathbb{E}\left\{\frac{K_{N-\alpha+1}(\beta \sqrt{\tau(t)})^{2}}{K_{N-\alpha}(\beta \sqrt{\tau(t)})^{2}}\right\} \frac{d \mathbf{m}(\theta, t)^{H}}{d \theta} \mathbf{M}^{-1} \frac{d \mathbf{m}(\theta, t)}{d \theta}
\end{aligned}
$$

in which the second step was done by noting that $\tau(t)$ has the same distribution as $z(\theta, t)$ and by using $\mathbf{n}(t)=\sqrt{\tau(t)} \mathbf{c}(t)$. The last step was done by noting that $\Re\left\{\mathbf{c}(t)^{H} \mathbf{M}^{-1} \frac{d \mathbf{m}(\theta, t)}{d \theta}\right\} \sim \mathcal{N}\left(\mathbf{0}, \frac{1}{2} \frac{d \mathbf{m}(\theta, t)^{H}}{d \theta} \mathbf{M}^{-1} \frac{d \mathbf{m}(\theta, t)}{d \theta}\right)$.

\section{III-C. Result and Gaussian Fisher information connec- tion}

The Gaussian Fisher information w.r.t. the model given in (1), i.e., with $\mathbf{n}(t) \sim \mathcal{C N}(\mathbf{0}, \mathbf{M})$, is given by the SlepianBang formula by

$$
\mathrm{FI}_{\mathrm{G}}(\theta)=2 \sum_{t=1}^{L} \frac{d \mathbf{m}(\theta, t)^{H}}{d \theta} \mathbf{M}^{-1} \frac{d \mathbf{m}(\theta, t)}{d \theta}
$$

Consequently, using the fact that $\Gamma(\alpha-1)=(\alpha-2) \Gamma(\alpha-2)$, (12) and (14), one deduces the link between the Gaussian FI, $\mathrm{FI}_{\mathrm{G}}(\theta)$, and the derived FI for K-distributed observations, $\operatorname{FI}(\theta)$ :

$$
\mathrm{FI}(\theta)=\Phi(\alpha, \beta, N) \mathrm{FI}_{\mathrm{G}}(\theta)
$$

in which

$$
\Phi(\alpha, \beta, N)=\frac{\beta^{2}}{4} \mathbb{E}\left\{\frac{K_{N-\alpha+1}(\beta \sqrt{\tau(t)})^{2}}{K_{N-\alpha}(\beta \sqrt{\tau(t)})^{2}}\right\} .
$$

It is worth noting that the multiplicative factor $\Phi(\alpha, \beta, N)$ is $\theta$ independent and depends only on the intrinsic gamma distribution's parameters and the size of the observation vector. This means that the previous computed CRLB for the Gaussian case can be directly used under the K-distributed observation just by numerically evaluating $\Phi(\alpha, \beta, N)$.

\section{EXTENSION TO THE MULTI-DIMENSIONAL CASE}

We assume, in the model (1), that the unknown parameter of interest $\boldsymbol{\theta} \in \mathbb{R}^{M}$, i.e., the parametric model is now given by $\mathbf{x}(t)=\mathbf{m}(\boldsymbol{\theta}, t)+\mathbf{n}(t), t=1, \ldots, L$. Due to the space limitation we present, in the following, only the result for the FIM. Nevertheless, we note that the methodology, tricks and derivations are exactly the same as in the scalar case.

$$
[\mathbf{F I}(\boldsymbol{\theta})]_{i, j}=\Phi(\alpha, \beta, N)\left[\mathbf{F I}_{\mathrm{G}}(\boldsymbol{\theta})\right]_{i, j}
$$


in which $\Phi(\alpha, \beta, N)$ in given by (16) and the Gaussian FIM w.r.t. $\boldsymbol{\theta}$, for $(i, j) \in\{1, \ldots, M\}^{2}$, is

$$
\left[\mathbf{F I}_{\mathrm{G}}(\boldsymbol{\theta})\right]_{i, j}=2 \sum_{t=1}^{L} \Re\left\{\frac{\partial \mathbf{m}(\boldsymbol{\theta}, t)^{H}}{\partial[\boldsymbol{\theta}]_{i}} \mathbf{M}^{-1} \frac{\partial \mathbf{m}(\boldsymbol{\theta}, t)}{\partial[\boldsymbol{\theta}]_{j}}\right\}
$$

\section{NUMERICAL ILLUSTRATION}

In this section we perform a numerical simulation to illustrate the previous study in the case of sensor array processing. Consider $M$ radiating far-field narrowband sources, from direction of arrival $\theta_{m}$ with $m=1, \ldots, M$ and $\lambda$ as wavelength, observed by a unifrom and linear array (ULA) composed of $N$ sensors with interelement spacing $d$. The parameterized mean is then given by $\mathbf{m}(\boldsymbol{\theta}, t)=\boldsymbol{A} \boldsymbol{s}(t)$, where the steering matrix is given by $\boldsymbol{A}=\left[\boldsymbol{a}\left(\theta_{1}\right), \ldots, \boldsymbol{a}\left(\theta_{M}\right)\right]^{T}$ in which $\left[\boldsymbol{a}\left(\theta_{m}\right)\right]_{n}=\exp ^{-2 \pi \frac{d}{\lambda} n \sin \left(\theta_{m}\right)}$ for $n=0, \ldots, N-1$ and the source signal vector is $s(t)=\left[s_{1}(t), \ldots, s_{M}(t)\right]$ in which the complex time-varying signal source for the $m$-th source is denoted by $s_{m}(t)$. In this scenario the unknown parameter vector is given by $\boldsymbol{\xi}=\left[\boldsymbol{\theta}, \mathcal{S}(\mathbf{M})^{T}\right]^{T} \in \mathbb{R}^{M+N^{2}}$. Furthermore, we consider the well known configuration, in the radar context, for which $\beta=\frac{1}{\alpha}$ (i.e., the case of a correlated heavy tailed clutter).

It is straightforward to derive the FIM using (17) and to deduce the CRLB for a fixed number of sources, for which an illustration is give in Fig. 1:

- This simulation reveals that for large $\alpha$ an efficient estimator under K-distributed noise exhibits the same performance than an efficient estimator in the classical case, i.e., in a Gaussian context. Whereas, for smaller $\alpha$ the variance of this latter degrades.

- From Fig. 1 we can note that the CRLB in the Kdistributed observation context for the parametrized mean is lower than the Gaussian one. This case has already been observed in the case of Gaussian mixture in [16]. Nevertheless, it should be noticed that this it not the case for the parametrized covariance matrix only case for which the opposite phenomenon is observed [9].

- Finally, from (16), one note that, for a fixed $\alpha$ and large number of sensors, an efficient estimator under K-distributed noise and an efficient estimator under Gaussian noise has a comparable variance.

Note that the second and third items were verified by simulation, but due to the lack of space, these simulations could not be added.

\section{CONCLUSION}

In this communication, an extension of the Slepian-Bang formula for the computation of the Fisher information is provided in the context of K-distributed observation. Semi closed form expression are provided for the general case of parameterized mean and the connection with the Gaussian model assumption is presented. Numerical simulation reveals that the CRLB in the K-distributed observation context for the parametrized mean is lower than the classical one. Nevertheless, it should be noticed that this it not the case for the parametrized covariance matrix.

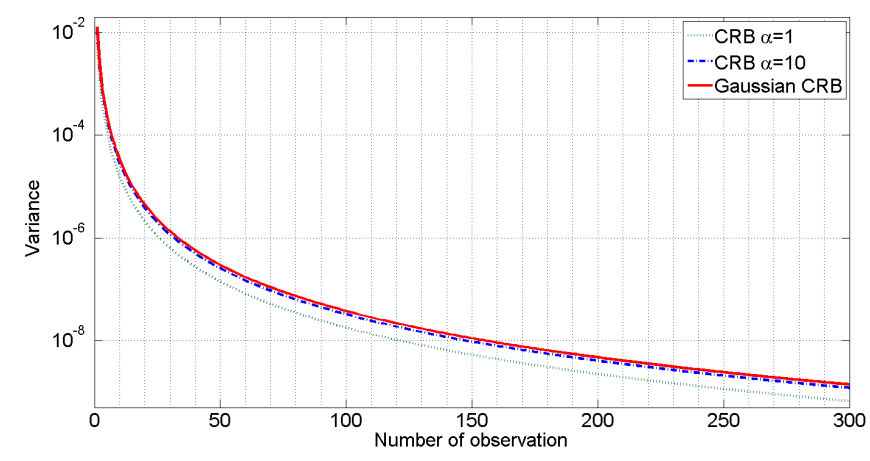

Fig. 1. The CRLB w.r.t. $\theta_{1}$ under K-distributed noise for different $\alpha$ and under the classical Gaussian noise. $\mathrm{N}=10$ and $\mathrm{M}=2$ with two orthogonal signal sources.

\section{REFERENCES}

[1] E. L. Lehmann and G. Casella, Theory of Point Estimation. New York: Wiley, 1998

[2] A. Renaux, P. Forster, E. Chaumette, and P. Larzabal, "On the high SNR conditional maximum-likelihood estimator full statistical characterization," IEEE Trans. Signal Processing, vol. 12, no. 54, pp. 4840-4843, Dec. 2006.

[3] S. M. Kay, Fundamentals of Statistical Signal Processing : Estimation Theory. NJ: Prentice Hall, 1993, vol. 1.

[4] J. B. Billingsley, "Ground clutter measurements for surface-sited radar," Massachusetts Inst. Technol., Cambridge, MA,, vol. Tech. Rep. 780, Feb. 1993.

[5] F. Gini and M. V. Greco, "Covariance matrix estimation for CFAR detection in correlated heavy tailed clutter," Signal Process., vol. 82, pp. 1847-1859, Dec. 2002.

[6] F. Pascal, Y. Chitour, J.-P. Ovarlez, P. Forster, and P. Larzabal, "Covariance structure maximum likelihood estimates in compound gaussian noise: Existence and algorithm analysis," IEEE Trans. Signal Process., vol. 56, pp. 34-48, Jan. 2008.

[7] D. Blacknell, "Comparison of parameter estimators for Kdistribution," IEE Proc. Radar Sonar Navigation, vol. 141, Jan. 1994.

[8] F. Gini, "A radar application of a modified Cramér Rao bound: parameter estimation in non-Gaussian clutter," IEEE Trans. Signal Process., vol. 46, pp. 1945-1953, Jul. 1998.

[9] F. Pascal and A. Renaux, "Statistical analysis of the covariance matrix MLE in K-distributed clutter," Elsevier Signal Processing, vol. 90, pp. 1165-1175, Apr. 2010.

[10] M. Greco and F. Gini, "Cramer-Rao lower bounds on covariance matrix estimation for complex elliptically symmetric distributions," IEEE Trans. Signal Process., vol. 61, pp. 6401-6409, Dec. 2013.

[11] J. Wang, A. Dogandzic, and A. Nehorai, "Maximum likelihood estimation of compound-Gaussian clutter and target parameters," IEEE Trans. Signal Processing, vol. 54, pp. 3884-3898, Oct. 2006.

[12] X. Zhang, M. N. El Korso, and M. Pesavento, "MIMO radar performance analysis under K-distributed clutter," in Proc. of IEEE Int. Conf. Acoust., Speech, Signal Processing, vol. 5, Firenze, Italy, 2014, pp. 5324-5328.

[13] O. Besson and Y. I. Abramovich, "On the Fisher information matrix for multivariate elliptically contoured distributions," vol. 20, pp. 11301133, Nov. 2013.

[14] K. Yao, "A representation theorem and its applications to spherically invariant random processes," IEEE Trans. Inf. Theory, vol. 19, pp. 600-608, Sep. 1973.

[15] M. Abramowitz and I. A. Stegun, Handbook of Mathematical Functions with Formulas, Graphs and Mathematical Tables. New York: Dover Publications, inc., 1972.

[16] B. M. Sadler, R. J. Kozick, and T. Moore, "Performance analysis for direction finding in non-Gaussian noise," ICASSP'99, vol. 5, pp. 2857-2860, May 1999. 